Voix et Images

\title{
Soirée bénéfice pour tous ceux qui ne seront pas là en l'an deux mille
}

\section{Dominique Lafon}

Volume 33, numéro 1 (97), automne 2007

Michel Marc Bouchard

URI : https://id.erudit.org/iderudit/017525ar

DOI : https://doi.org/10.7202/017525ar

Aller au sommaire du numéro

Éditeur(s)

Université du Québec à Montréal

ISSN

0318-9201 (imprimé)

1705-933X (numérique)

Découvrir la revue

Citer cet article

Lafon, D. (2007). Soirée bénéfice pour tous ceux qui ne seront pas là en l'an deux mille. Voix et Images, 33(1), 27-29. https://doi.org/10.7202/017525ar d'utilisation que vous pouvez consulter en ligne.

https://apropos.erudit.org/fr/usagers/politique-dutilisation/ 


\title{
SOIRÉE BÉNÉFICE POUR TOUS CEUX QUI NE SERONT PAS LÀ EN L'AN DEUX MILLE
}

\author{
DOMINIQUE LAFON
}

Université d'Ottawa

Soirée bénéfice pour tous ceux qui ne seront pas là en l'an 2000 pourrait bien être la pièce qui ne sera jamais dans la bibliographie des œuvres de Michel Marc Bouchard, même si elle a pourtant fait à ce jour l'objet de six productions ou lectures publiques: trois mises en «lecture-spectacle» dirigées par Brigitte Haentjens à Ottawa en 1989 et 1990, puis, l'année suivante, à Toronto, suivies d'une lecture par une compagnie belge à Limoges en 1991 et de deux productions, sous deux versions différentes, mises en scène par Gil Champagne en mai et août 1992 à Québec. Voilà donc quinze ans que cette pièce est ignorée du public comme de la critique qui n'a pas accès à ses cinq ou peut-être six versions dont l'auteur m'a confié la conservation et l'usage. C'est aussi avec sa permission que le présent dossier en donne à lire et à voir quelques extraits.

Je dois ce statut de dépositaire, bien sûr, à une longue familiarité avec l'écriture de Michel Marc Bouchard, mais aussi, et surtout, aux circonstances qui ont entouré la naissance de cette pièce qu'il conçut alors qu'il était, à Ottawa, directeur artistique du Thêâtre d'la Corvée qu'il s'empressa de rebaptiser sous le nom de Thêâtre du Trillium, nom sous lequel il est encore connu aujourd'hui. Cette direction artistique fut douce à notre amitié puisqu'elle abolissait, quelques jours par semaine, la distance qui sépare Montréal de la capitale fédérale. C'est donc tout naturellement que je fus conviée à me joindre non pas à « une entreprise qui n'eut jamais d'exemple et dont l'exécution n'aura point d'imitateur ${ }^{1} "$, mais à des rencontres qui font désormais partie de tous les works in progress de la modernité, au cours desquelles le dramaturge veut éprouver, réviser, commenter avec les comédiens et le metteur en scène une œuvre déjà écrite... sous le bienveillant regard d'une équipe de télévision.

Quatre versions furent ainsi conçues dans un jeu de remaniements qui étaient autant de concessions aux participants qui ne laissaient pas leur place: Brigitte Haentjens, Robert Bellefeuille, Robert Marinier et Anne-Marie Cadieux n'étaient pas encore au pinacle de leur carrière, mais ils montraient déjà de belles prédispositions

$$
+++
$$

1 Jean-Jacques Rousseau, Les confessions, Fuvres complètes, t. I, sous la direction de Bernard Gagnebin et Marcel Raymond, Paris, Gallimard, coll. «Bibliothèque de la Pléiade», 1959, p. 5. 
au vedettariat. Ce fut donc toute une aventure qui se solda par une sorte de compromis étrange selon lequel les comédiens en représentation lisaient le texte (avec lutrins), mais utilisaient aussi l'espace au centre duquel trônait, le mot n'est pas trop fort, une vraie grosse voiture dont l'installation coûta une petite fortune.

Voilà pour l'anecdote et les souvenirs.

Il faut dire que la voiture est essentielle à une pièce dont la situation initiale est provoquée par une panne au bord de la route au beau milieu d'une forêt qui, à défaut d'être profonde (elle se limite à une mince rangée d'arbres), semble déserte. C'est le 31 décembre 1999 et les étoiles filent dans le ciel comme pour annoncer une imminente apocalypse. La famille dont les membres sortent les uns après les autres de la voiture est, elle aussi, en panne ou, pour prendre un terme qui fait florès en psychologie, totalement dysfonctionnelle. Les fils Bruno, Martin et Pierre-Louis sont d'une agressivité extrême à l'égard de leur mère Mathilde, présidente des Fourrures Mercier, alors que Lili, la fille de Bruno, se mure dans un mutisme hostile. Deux personnages secondaires complètent la scène domestique, deux femmes au service de la famille: Nathalie, employée d'une agence d'escorte qui sert de «couverture » à Pierre-Louis pour dissimuler son homosexualité, et Flora, une violoncelliste brésilienne, souvenir de voyage de Mathilde.

Dans les premières versions, une mise en abyme se dessine, donnant à croire que toute la pièce est un fantasme de Lili, l'actualisation de son journal intime dans lequel elle imagine une révolte des enfants, une insurrection planétaire qui oblige les parents à fuir leur progéniture ou à l'exterminer avant qu'elle ne leur fasse un mauvais sort. En attendant, les enfants regroupés en hordes sauvages brûlent et saccagent tout sur leur passage. Cet imaginaire vengeur est inspiré à Lili par un traumatisme de sa propre enfance qui s'est produit dix ans plus tôt alors qu'elle a vu, du haut de l'escalier menant à sa chambre, son père Bruno battre violemment Nathalie, sa mère. Celle-ci, prise de boisson, lui avait annoncé qu'elle était atteinte d'une maladie mortelle transmise par un amant de passage. La référence au sida est implicite, mais ce n'est pas la seule évocation des maux qui frappent ce monde en furie: pêle-mêle sont évoqués la misère du Tiers-Monde (par le biais de Flora), les désastres écologiques, le cancer (Pierre-Louis a une maladie de la peau) et la maladie d'Alzheimer (Mathilde doit réciter son alphabet pour entraîner sa mémoire). L'apocalypse imaginaire se nourrit d'actualité réelle et d'une «légende judaïque, la Gouve», qui expliquerait prophétiquement l'insurrection: «La Gouve, c'est le réservoir des âmes. Ã chaque fois qu'un enfant naît, La Gouve se vide d'une âme mais il paraît qu'il y a un nombre limité d'âmes. La Gouve serait à sec. La génération de Lili aurait pas d'âme. Ce serait la génération de la violence, la dernière génération. C'est un des signes avant-coureurs de l'Apocalypse.»

Dans la dernière version représentée, dont sont reproduites ici les cinq premières scènes ${ }^{2}$, cette légende empruntée est remplacée par une légende inventée, celle du père-loup, qui modifie complètement le sens de la pièce. Le personnage de

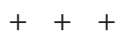

2 Ce document est précédé d'extraits des trois premières versions qui permettent de suivre le travail de réécriture. 
Lili a été supprimé et Nathalie est devenue la sœur sans nom de la famille stérile condamnée à l'immortalité. Ce mythe personnel est opératoire puisque c'est lui qui explique les conflits internes d'une cellule déchirée par son rapport à la mère qui sera mise à mort par Martin, le fils qu'elle croit avoir séduit en le choisissant pour succéder à Pierre-Louis, malade. La dimension incestueuse est explicite, qui sous-tend les relations entre la mère et ses fils, comme celles entre la sœur et ses frères. La panne n'est plus une panne sèche, mais un accident délibérément provoqué par Bruno qui, comme Victor dans Le chemin des Passes-dangereuses, veut soumettre sa famille au jugement dernier, en l'occurrence celui du millénaire. Ce paradoxal huis clos en pleine nature, une nature dévastée par l'homme, est complet puisque Flora, la violoncelliste, a été engagée par Mathilde pour donner des lambeaux de sa peau à Pierre-Louis qui a besoin de greffes.

Ce huis clos est sans issue; aucune violence, aucune apocalypse n'en délivrera ces lycanthropes qui ne peuvent mourir, à la différence des loups de l'élevage des Mercier qui, dans les premières versions, avaient tous succombé à une étrange épidémie et ruiné du coup l'entreprise. Si la famille québécoise était sortie de la cuisine où la dramaturgie des années soixante-dix l'avait enfermée, elle n'était pas pour autant sortie du bois... et la pièce de Michel Marc Bouchard le faisait bien voir qui la vouait aux gémonies d'une Histoire sans fin. 